Erschienen in: Stickel, Gerhard (Hrsg.): Varietäten des Deutschen. Regional- und Umgangssprachen. - Berlin, New York: de Gruyter, 1997. S. 221-245. (Institut für deutsche Sprache. Jahrbuch 1996)

\title{
MATTHIAS WERMKE
}

\section{Umgangssprachliches im standardsprachlichen Wörterbuch des Deutschen}

\begin{abstract}
Der Beitrag versucht zunächst, den linguistischen Beschreibungsgegenstand Umgangssprache auf der Grundlage der neueren Forschungen hierzu näher zu spezifizieren. Es geht dabei um die Frage, welche sprachlichen Erscheinungen als umgangssprachlich bewertet werden und wie Umgangssprache allgemein definiert wird. Daraus resultiert die Feststellung, daß hierüber in der Forschung kein Konsens besteht und der Terminus Umgangssprache durchaus mehrdeutig verwendet wird. Dieser Tatbestand wirkt sich unmittelbar auf die lexikographische Praxis aus. Hier fehlt es an einem klaren Konzept der zu differenzierenden Stilschichten, hier mangelt es auch an einheitlichen Markierungskriterien und an einer einheitlichen Markierungspraxis. Divergenzen werden erklärt als Folge zwangsläufig empirischer, auf dem Sprachgefūhl des einzelnen Lexikographen beruhender Zuordnungen. Am Beispiel des Deutschen Universalwörterbuches und anderer allgemeiner Wörterbücher des Gegenwartsdeutschs wird gezeigt, welche umgangssprachlichen Elemente im Wörterbuch überhaupt behandelt werden und welche Schwierigkeiten es insbesondere im Hinblick auf die regionale Gebundenheit umgangssprachlicher lexikalischer Einheiten gibt.
\end{abstract}

\section{Umgangssprache als Gegenstand wissenschaftlicher Beschreibung}

Eine klar umrissene Vorstellung davon, was unter Umgangssprache eigentlich zu verstehen ist, scheint es bei vielen Sprachteilhabern nicht zu geben. Jedenfalls löst die dahingehende Frage bei den meisten der hierzu Befragten sichtliche Irritation aus. Entsprechend vorsichtig sind die Antworten: Umgangssprache? Das ist so, wie wir halt reden; oder: So reden wir, wenn wir einander verstehen wollen; sehr häufig wird der Bezug zum Dialekt hergestellt: Umgangssprache? Wenn wir Dialekt sprechen, dann ist das Umgangssprache.

Sicherlich sind solche Spontanbefragungen nicht repräsentativ. Sie geben aber immerhin ein Indiz dafür, daB der so geläufige Begriff Umgangssprache offenbar nicht leicht einzugrenzen ist. Das gilt für die Laien, das gilt aber auch in der Linguistik, wo er ebenfalls keineswegs fest umrissen ist. Es fehlt eine einheitliche und allgemein anerkannte Definition. Es fehlt eine eindeutige Abgrenzung des Untersuchungsgegenstandes. Unklar ist, ob das, was mit dem "vorwissenschaftlichen Terminus" (Bichel 1980, S. 379) Umgangssprache bezeichnet wird, als eigene Varietät des Deut- 
schen zu werten ist, oder ob es sich dabei eher um ein Phänomen des Stils handelt. Diese Unentschiedenheit kann sich auf die lexikographische Praxis auswirken und führt zum Beispiel dazu, daß ein bestimmtes Lexem im einen Wörterbuch als landschafilich markiert ist (so. z. B. „ruschelig” im Rechtschreibduden), im anderen aber als umgangssprachlich (so im einbändigen Deutschen Wörterbuch von Wahrig).

Beim Vergleich der unterschiedlichen Definitionsansätze kristallisieren sich immerhin vier Hauptkriterien heraus, durch die Umgangssprache im allgemeinen bestimmt wird. Es sind dies das Kriterium der Mündlichkeit, das Kriterium der sozialen Gebundenheit, das Kriterium der räumlichen Gebundenheit und das Kriterium der stilistischen Gebundenheit.

\subsection{Das Kriterium der Mündlichkeit}

Was die Umgangssprache nach vielfach vertretener Ansicht besonders auszeichnet, ist ihre funktionale Beschränkung auf den mündlichen Gebrauch. Dies bestätigt auch die eingangs zitierte Umfrage. Herbert E. Wiegand faBt den neueren Forschungsstand zusammen und sagt, es bestehe Einigkeit darüber, daß "Umgangssprachen Sprechsprachen" seien und meistens als „Interferenzvarianten” aufgefaBt würden, „die auf allen sprachlichen Ebenen aus anderen Varietäten (wie Fachsprachen, Berufssprachen, speziellen Gruppensprachen und Dialekten) und aus der zugehörigen Leitvarietät übernehmen" (Wiegand 1989, S. 2202).

Bei Rosemarie Lühr ist Umgangssprache „die mündliche Sprachverwendung im Wechsel mit einem oder mehreren Gesprächspartnern” bzw. „die Varietät (sämtliche für eine Region, Gruppe oder Situation typische Varianten) einer Sprache, die im Gespräch, in mündlicher Kommunikation, typisch ist" (Lühr 1990, S. 242).

Ähnlich Ulf Bichel. Er unterscheidet „primäre Umgangssprache” als "Sprachvarietät in Gruppen mit unmittelbarem persönlichen Kontakt" das bedeutet Face-to-face-Kommunikation und demzufolge in aller Regel Mündlichkeit - und „sekundäre Umgangssprache” als "Sprachvarietät, die im Zwischengruppenverkehr üblich ist" (Bichel 1980, S. 381). Offen bleibt, inwieweit im letzten Falle ggfs. auch an Schriftlichkeit zu denken wäre.

Nach Lewandowski ist Umgangssprache "die unvorbereitet-spontane Rede von Sprechern der Standardsprache bei direkter nicht-offizieller Kommunikation" bzw. "die städtische und ländliche Alltagsrede” (Lewandowski 1976, S. 853). Wichtig hierbei ist, daB Umgangssprache als eine besondere gesprochene Form der Standardsprache gilt, wobei eine situative Komponente in den Vordergrund tritt - "nicht-offizielle Kom- 
munikation" -, die bei der Beantwortung der Frage, wie man Umgangssprachliches im Wörterbuch behandeln könnte, von Bedeutung sein kann.

Die allgemeinen Nachschlagewerke und die Wörterbücher greifen diese Definitionen auf. Mündlichkeit ist das hervorstechende Merkmal, wenn die Brockhaus-Enzyklopädie Umgangssprache bestimmt als "Sprache, die im täglichen Umgang mit anderen Menschen verwendet wird (Alltagssprache)" bzw. als "nachlässige, saloppe bis derbe Ausdrucksweise" bzw. als "Sprache, in der eine Gruppe miteinander umgeht, sich unterhält” (BE 22, S. 593). Im Deutschen Wörterbuch von Brockhaus-Wahrig gilt Umgangssprache als „Sprache, die im täglichen Umgang (= gesellschaftlicher Verkehr) gesprochen wird", als nSprachebene zwischen Hochsprache und Mundart, die bei nichtöffentlicher Kommunikation verwendet wird" (BW 1984, Bd. 6, S. 366). Das GroBe Wörterbuch der deutschen Sprache (GWB) und das Deutsche Universalwörterbuch (DDUW) definieren Umgangssprache schlieBlich (1a) als ${ }_{n}$ Sprache, wie sie im täglichen Umgang mit anderen Menschen verwendet wird; zwischen Hochsprache und Mundart stehende, von regionalen, soziologischen, gruppenspezifischen Gegebenheiten beeinflußte Sprachschicht" bzw. (1b) als "nachlässige, saloppe bis derbe Ausdrucksweise; Slang” und (2) auch als "Sprache, in der eine Gruppe miteinander umgeht, sich unterhält" (GWB ${ }^{2} 1995$, S. 3521 u. DDUW ${ }^{2} 1989$, S. 1598).

Gegen diesen ausschließlichen Primat der Mündlichkeit hat sich Hugo Steger gewandt und kritisiert, daB "die Forschung entschieden zu kurz" greife, "wenn sie den Bereich der Alltagskommunikation als einzigen funktionalen Bereich" ansehe, ,in dem die Umgangssprache eingesetzt" werde „und etwas leisten" müsse (Steger 1984, S. 225). Er bemerkt, daf sie nicht nur "in gesprochener, sondern auch in geschriebener Form" vorkomme (a.a.O., S. 257). Dies läBt sich an Hand von Textbelegen nachweisen, und zwar gerade an solchen, in denen nichtgesprochene Sprache zitiert wird. So liest man im „Spiegel”, Heft 46/1975 zum Beispiel "Oder sie aalten sich in Miami-Beach im lauen Atlantik ..." (a.a.O., S. 149). In der "Welt" vom 20.7.1965 ist "Jeder bimste sich ein, was er aufgeschnappt hat" belegt (a.a.O., S. 7), und in der "Neuen Zürcher Zeitung" gibt es am 9.12.1982 für die Schweizerischen Bundesbahnen "nur eine Möglichkeit, aus dem Schlamassel herauszukommen ..." (a.a.O., S. 27). Willy Brandt schlieflich konnte ${ }_{n}$ die kaltschnäuzige Art nicht leicht vergessen, mit der gewisse Verbandsvertreter [seine] Warnungen vom Tisch fegten" (W. Brandt, Begegnungen und Einsichten 1976, S. 582). „[sich] aalen", "[sich] einbimsen”, "Schlamassel" und "kaltschnäuzig" sind im DDUW alle als umgangssprachlich markiert. So übrigens auch im Rechtschreibduden, dem Deutschen Wörterbuch von Brockhaus-Wahrig und 
anderen gegenwartssprachlichen Wörterbüchern des Deutschen. Sämtliche Belege stammen aus der Sprachkartei der Dudenredaktion.

\subsection{Das Kriterium der sozialen Gebundenheit}

DaB Umgangssprache auch als gruppenspezifisches Phänomen betrachtet wird, klang in den zitierten Definitionen bereits an. Im Hinblick auf ihre soziale Gebundenheit setzt sich heute jedoch mehr und mehr die von Ingo Reiffenstein schon 1968 konstatierte Einsicht durch, daB Umgangssprache „sozial-indifferent” sei, d.h. - wie Hugo Steger ergänzt daB sie "von fast allen gesellschaftlichen Gruppen/Schichten/Klassen der deutschsprachigen Gesellschaften im Rahmen situationstypischer Normen benutzt" wird (Steger 1984, S. 259). Umgangssprache, so könnte man auch ein wenig salopp sagen, ist heute "salonfähig". Nicht von ungefähr spielt sie in den Wörterbüchern eine gewisse Rolle, was bei Sprachpuristen durchaus auf Kritik stößt. Ihr Gebrauch ist eher situationsbedingt und weniger gruppenspezifisch. Umgangssprachensprecher sind wir alle.

\subsection{Das Kriterium der räumlichen Gebundenheit}

Die Einordnung der Umgangssprache in das Varietätenspektrum des Deutschen ist nicht einfach. Für sie gilt, daB sie "keine klar abgrenzbare Sprachvarietät" ist (Wolski 1995, S. 348). So bezeichnet der Terminus Umgangssprache eben auch "den großen und heterogenen Bereich von Sprachvarietäten zwischen Hochsprache/Standardsprache einerseits und kleinräumig gebundenen Dialekten andererseits" (Bußmann 1990, S. 814). Sie wird aufgefaBt als eine Art von "Ausgleichsvarietät zwischen Hochsprache und Dialekt, die zwar deutlich regionale Färbung, jedoch keine extremen Dialektismen aufweist" (ebenda). Ähnlich definiert schon Ingulf Radtke 1973. Bei ihm ist Umgangssprache die "gesprochene deutsche Sprache ..., die überregional gesprochen und verstanden wird, nicht fachgebunden (Fachsprache) und nicht verhüllend (Sondersprache) ist, aber durchaus landschaftliche Züge (etwa in den Intonationsverhältnissen) aufweisen kann" (Radtke 1973, S. 170). Das Zugeständnis einer „regionalen Färbung” oder „landschaftlicher Züge” ist prinzipiell etwas anderes, als die in der jüngeren Forschung vermehrt vertretene Ansicht, daB Umgangssprache auch eine regionale Beschränkung habe. So bemerkt Helmut Henne im Hinblick auf die Markierung umgangssprachlich im Wörterbuch, sie signalisiere, daß der solchermaßen markierte Wortschatz "unterhalb der normalen Stilschicht” liege und „oft nur regionale Geltung" habe (Henne in Duden Bd. 4, 1995, S. 586). Entsprechend die Bedeutungsangabe in seiner Bearbeitung des Deut- 
schen Wörterbuchs von Hermann Paul, wo unter (2) Umgangssprache definiert wird als „sprachliche Existenzform zwischen Hochsprache und Mundart mit regionaler Geltung" (Henne 1989, S. 823). Auch Ulf Bichel verweist auf „areal fixierbare umgangssprachliche Erscheinungen” (Bichel 1980, S. 382), und Hugo Steger faßt Umgangssprache als einen "dritten Varietätentypus, der kommunikativ eine regionale Reichweite besitzt" (Steger 1984, S. 251) auf. Nicht von ungefähr hat sich - auch darauf weist er hin - der Begriff der "regionalen Umgangssprache" eingebürgert.

\subsection{Das Kriterium der stilistischen Gebundenheit}

Schlieblich wird der Terminus Umgangssprache auch als ${ }_{n}$ Bezeichnung einer Stilschicht, die für informellere, private Situationen angemessener erscheint als die eher auf formelle Situationskontexte beschränkt bleibende Hochsprache" (BuBmann 1990, S. 814) verwendet. Bei Henne war vom "unterhalb der normalen Stilschicht liegenden Wortschatz" die Rede (s. o.). Im Gegensatz zum Stil als bewußt geformter Sprache (vgl. hierzu: Bichel 1973, S. 333), wird Umgangssprache als ,ungeformte (d. h. ungewählte, unbekümmerte, ungepflegte oder nachlässige) Sprache" dargestellt (Bichel, ebenda). Umgangssprachlich ist all das, was als „schriftsprachlich nicht ganz korrekt” zu werten ist (Bichel 1973, S. 159).

Man kann also festhalten: Umgangssprache gilt heute zum Teil noch immer als ein Phänomen vorwiegend der gesprochenen Sprache. Sie wird aber - gegen die Meinung der älteren Forschung - nicht nur von Vertretern bestimmter sozialer Gruppen verwendet, sondern von Mitgliedern aller gesellschaftlichen Gruppen und Schichten in Abhängigkeit vom Kommunikationskontext. Umgangssprache kann regional geprägt sein und hat zum Teil areal fixierte Verbreitung; demzufolge ist also nicht die Umgangssprache, sondern eine unbestimmte Menge von Umgangssprachen anzusetzen, was ihre Beschreibung zusätzlich erschwert. Umgangssprachliches gilt noch vielfach in eher offiziellen Kommunikationskontexten als anstößig, von der Standardnorm negativ abweichend. Als besondere Charakteristika der Umgangssprache nennt Rosemarie Lühr zusammenfassend auf der Ebene der Syntax „die Neigung zu kurzen Sätzen, zur Nebenordnung, zum Einschub von Interjektionen und zu Freiheiten des Satzbaus...”, im Bereich der Phonologie „Kontraktionen ... und weitere Lässigkeiten der Aussprache" und im Bereich der Lexik „der Gebrauch von Allerweltswörtern wie machen, tun, Ding" (Lühr 1990, S. 242). Steger weist zusätzlich darauf hin, daß „standardsprachliche Tabubereiche ... entsprechend der tatsächlichen Bedeutung solcher Felder für die allgemeine Lebenspraxis und auf Grund des breiteren Nor- 
menspektrums der Benutzergruppen der Umgangssprache in der Lexik der Umgangssprache reich ausgebildet" seien (Steger 1984, S. 253). Nicht zu vergessen sind schließlich ganz allgemein Verstöße gegen die normativen Regeln, wie zum Beispiel der Gebrauch des Dativs bei wegen oder fehlende Inversion in der Satzstellung nach weil. Dem Thema dieses Beitrags entsprechend, wird im folgenden nur der lexikalische Aspekt näher betrachtet werden.

\section{Die Dokumentation umgangssprachlicher Lexik im allgemeinsprachlichen Wörterbuch}

\subsection{Die Differenzierung von Stilschichten}

Die allgemeinsprachlichen Wörterbücher des Deutschen machen es sich zur Aufgabe, "die deutsche Sprache in ihrer ganzen Vielschichtigkeit zu dokumentieren", wie es zum Beispiel im Vorwort des Großen Wörterbuches der deutschen Sprache heiBt (GWB ${ }^{2} 1993$, Vorwort), bzw. „die Vielfalt der deutschen Sprache darzustellen und zu beschreiben", wie im Brockhaus-Wahrig nachzulesen ist (BW 1980, S. 5). Dieser Anspruch zwingt dazu, neben dem standardsprachlichen Wortschatz auch areale Varianten, fach- und sondersprachliches Wortgut und eben auch derartige lexikalische Einheiten zu berücksichtigen, die eine „unmittelbare Auffäligkeit" (Hausmann 1989, S. 649) haben. Eine solche Auffalligkeit ergibt sich, so Ludwig (1995, S. 297) "durch ein gegenüber der Normalität abweichendes Merkmal, das der sprachlichen Einheit wie ein Etikett anhaftet." DaB es die Aufgabe der Lexikographie ist, diese zusätzlichen Merkmale im Wörterbuch auszuweisen, ist unumstritten. Umstritten ist allerdings, wie dieses am sinnvollsten geschieht.

Zunächst besteht das Problem, daB es innerhalb der Lexikographie hinsichtlich der pragmatischen Markierungen kein einheitliches Beschreibungsinventar gibt (vgl. dazu Ludwig 1991, S. 221). Hinzu kommt, daß in den Wörterbucheinleitungen aus linguistischer Sicht oft keine dezidierten oder nur unscharfe Aussagen zur Bedeutung verwendeter Marker gemacht werden. Dies ist in der Wörterbuchkritik verschiedentlich bemängelt worden (vgl. z. B. Hausmann 1989, S. 650; Ludwig 1995, S. 294 f.). Die Kritik richtet sich vor allem auch gegen das Fehlen expliziter Angaben zum Gebrauch des Markers ugs., der in der Wörterbuchpraxis im allgemeinen $z u$ den stilistischen Bewertungen gerechnet wird. Nun kann man aber durchaus unterschiedlicher Meinung darüber sein, ob es in einem Gebrauchswörterbuch generell nötig ist, ein Modell der angesetzten Stilschichten zu entwerfen und darzustellen. Es ist auch mehr als fraglich, ob Wörterbuchbenutzungshinweise oder Wörterbucheinleitungen der rechte Ort sind, ggfs. entsprechende Abgrenzungen zu an- 
deren Auszeichnungsmodellen vorzunehmen, was dann durchaus nötig wäre. Die Erfahrung zeigt, daB die Mehrzahl der Wörterbuchbenutzer offensichtlich keinen AnstoB an der herkömmlichen Auszeichnungspraxis nimmt. Es sind diesbezüglich jedenfalls kaum Reaktionen zu verzeichnen, und wenn, dann beziehen sie sich meistens auf diatopische Auszeichnungen und nicht auf stilistische. Man darf annehmen, daB diese offenkundige Akzeptanz daher rührt, daB Marker wie dichterisch, salopp, vulgär u. a. und natürlich auch Fachbereichsangaben wie Eisenbahnwesen, Kunstwissenschaft, Handwerk und viele andere selbstsprechend genug sind, um ihre Auszeichnungsfunktion erfüllen zu können. Hier gibt es seitens der Metalexikographie Anforderungen, die über das hinausgehen, was im Alltagsgebrauch als nötig empfunden wird. Nichtsdestotrotz sollte in den Wörterbucheinleitungen die Bedeutung der verwendeten Marker erläutert werden, was ja aber in der Regel durchaus geschieht.

Die Auszeichnung ugs. ist auch hinsichtlich ihrer Verwendung in Wörterbüchern mehrdeutig. Da kein einheitliches Konzept dessen, was als Umgangssprache zu beschreiben ist, vorliegt, müssen die "gegenwartsbezogenen Wörterbücher zwischen den verschiedenen Begriffen von 'Umgangssprache' vermitteln" (Henne 1988, S. 820). Nach Henne verweisen "Umgangssprache und das Adjektiv umgangssprachlich auf eine Stilschicht der Gemeinsprache; eine regionale Kennzeichnung auf lexikalische Lücken der Gemeinsprache, die durch Umgangssprachen gefüllt werden" (Henne, ebenda). Dennoch berühren sich beide Markierungen in vielen Fällen. In Wörterbüchern wird schlieBlich auch nur regional verbreiteten Lexemen ein Stilniveau umgangssprachlich zugeordnet. Beispiele hierzu aus dem DDUW sind die Lemmata "Hudeler” (landsch. ugs.), „Modder” (nordd. ugs.) und „unterschlupfen” (südd. ugs.). Wenn man aber Umgangssprache wie Henne als Stilschicht der Gemeinsprache auffaBt, dann wäre zu überlegen, ob es nicht sinnvoll sein könnte, für vergleichbare Phänomene bei arealen Varietäten des Deutschen zur besseren Unterscheidung eigene Marker zu setzen. DaB dies die Auszeichnung in der Praxis und ggfs. auch die Wörterbuchbenutzung nicht einfacher machen würde, ist einleuchtend.

Nun bleibt aber gelegentlich vage, was eigentlich unter einer Stilschicht - auch der Begriff Stilebene taucht auf - und ggfs. unter Stilfärbung zu verstehen ist. Einige Wörterbücher verzichten ganz auf eine Erläuterung der durchaus zahlreich vorhandenen stilistischen Bewertungen, was in jüngerer Zeit in der Metalexikographie besonders von Klaus-Dieter Ludwig kritisiert wurde. Wenn sich die Lexikographie mit dem Begriff des Stils bzw. der Stilschicht einerseits schwertut, so ist andererseits dennoch festzuhalten, daB diesbezügliche Benutzerhinweise doch relativ ge- 
nau den kommunikativen Verwendungsrahmen der mit einer bestimmten Angabe markierten Lemmata beschreiben und damit tatsächlich das leisten, worum es bei stilistischen Markierungen eigentlich geht. Stilistische Angaben haben nicht nur dokumentative Funktion, sondern sind als stark komprimierte Wortverwendungsanleitungen aufzufassen. Wenn im DDUW „Mistvieh”, „Säufer" oder „Schweinerei” als derb ausgezeichnet sind, dann nicht deshalb, weil diese Wörter an sich derb wären, sondern weil sie in bestimmten Kommunikationskontexten vermieden werden sollten. So heiBt es zum Beispiel in den Vorbemerkungen zur "Anlage und zum Artikelaufbau" im DDUW zu den mit geh. (= gehoben) ausgezeichneten Wörtern, daß sie "bei feierlichen Anlässen und gelegentlich in der Literatur $v$ e $r$ w e n $d$ e t" (Herv. vom Verf.) werden (DDUW ${ }^{2} 1989$, S. 9). Ähnliches gilt in bezug auf die Umgangssprache, zu der es heißt, daB sie „im alltäglichen, besonders im familiärvertraulichen, mündlichen Verkehr der Menschen untereinander üblich" sei "und in Briefen verwendet" werde (ebenda). Problematisch ist bei diesen Ausführungen allein, daB auch hier die Vorstellung eines Schichtenmodells durchscheint, wenn es vom bildungssprachlichen, gehobenen und dichterischen Wortgut heift, es stehe „oberhalb” der normalsprachlichen Wörter, während die Umgangssprache etc. „unterhalb” der normalsprachlichen Stilschicht stehe. Angesichts der Sprachwirklichkeit, in der umgangssprachliches Wortgut auch in offiziellen Kommunikationssituationen weniger und weniger stigmatisiert ist - man vergleiche die oben zitierten Belege -, und auch angesichts der Tatsache, daB keine klare Vorstellung davon besteht, was eigentlich eine Stilschicht ist und wie sie sich von anderen abgrenzen läßt, wäre zu überlegen, ob ein solches Modell zur Beschreibung eben dieser Sprachwirklichkeit noch genügt.

So schlägt Klaus-Dieter Ludwig neuerdings vor, statt von Stilschichten bzw. Stilebenen und zusätzlichen Stilfärbungen von der „kommunikativen Prädisposition" der Lexeme auszugehen. Eine lexikalische Einheit ist prädisponiert, wenn sie nur "in bestimmten Bereichen der Kommunikation gebraucht wird" (Ludwig 1995, S. 297). „Lexikalische Einheiten, die dieselbe kommunikative Prädisposition aufweisen, gehören dann zur selben kommunikativen Prädispositionsebene", die den rallgemeinen $\mathrm{Be}$ reich der Kommunikation" angibt, ,in dem die entsprechende lexikalische Einheit vorwiegend gebraucht werden kann" (Ludwig, ebenda). Eine lexikalische Einheit, die keine Auffäligkeit im Sinne Hausmanns zeigt, wäre demnach neutral; sie hätte im Wörterbuch eine Nullmarkierung, womit dem Wörterbuchbenutzer angezeigt ist, daB er sie in den meisten Kommunikationskontexten verwenden kann. Davon abgehoben wären dann bei Ludwig solche Einheiten, die „über neutral”, und solche, die „unter neutral" bewertet sind. Damit schlägt er hinsichtlich der situationsspe- 
zifischen Auszeichnungen in Wörterbüchern zunächst ein dreistufiges Grobmodell vor, für dessen unterste Stufe er jedoch zu erwägen gibt, diese ${ }^{2} \mathrm{u}$ differenzieren in 'umgangssprachlich' bzw. 'ungezwungen' und 'derb'" (Ludwig 1995, S. 297f.). Gegenüber dem - zugegebenermaben etablierten, dafür aber mehrdeutigen - Etikett umgangssprachlich hätte die Markierung ungezwungen den Vorteil, daB sie sich eindeutiger auf den Kommunikationsrahmen beziehen lieBe. Bei derb ist dies alierdings schon nicht mehr der Fall. AuBerdem muB angemerkt werden, daB die vorgeschlagenen Werte über neutral - neutral - unter neutral für den durchschnittlichen Wörterbuchbenutzer sehr abstrakt sind und deshalb ggfs. als Markierungen im Gebrauchswörterbuch nicht geeignet sind.

Aber auch Ludwig bleibt bei seinem Vorschlag dem traditionellen Stufenmodell verhaftet. Wenn sein Ansatz insgesamt weniger, dafür aber vielleicht besser abgrenzbare Stufen hat, dann mag dies die lexikographische Auszeichnungspraxis zwar erleichtern. Es wird aber nicht dem bereits erwähnten Tatbestand gerecht, daB das, was im allgemeinen als umgangssprachlich charakterisiert wird, heute in weiten Kommunikationsbereichen anzutreffen ist, die vormals eher von der Standardsprache besetzt waren. Sein Ansatz führt letztlich nicht wirklich weg vom herkömmlichen hierarchischen Modell der Stilschichten, die auf einer Skala von dichterisch bis hinunter zu vulgär angesiedelt sind und entsprechend bewertet werden. Wenn man von der kommunikativen Prädisposition der Wörter ausgeht, dann sollte man nicht kommunikative Prädispositionsebenen, sondern kommunikative Prädispositionsbereiche ansetzen, womit ein wertfreier Begriff gewählt wäre, der eher deren Nebeneinander ausdrückt als deren hierarchische Stufung. Man könnte dann die ebenfalls wertende Dreigliederung neutral - über neutral - unter neutral ersetzen durch die Drias allgemeinverwendbar und folglich mit Nullmarkierung im Wörterbuch - literarisch und ungezwungen. Der kommunikative Prädispositionsbereich allgemeinverwendbarer Wörter könnte das herkömmlich als bildungssprachlich ausgewiesene Wortgut teilweise mit einschlieBen, zu einem anderen Teil liebe es sich wohl auch den Fachsprachen zuordnen. Entsprechende Tendenzen sind in den Wörterbüchern schon deutlich zu erkennen. Als Beispiele aus dem Deutschen Universalwörterbuch seien nur die Fälle „abundant” (bildungsspr., Wissenschaftsspr.), "Abusus" (bildungsspr., auch Med.), "akkumulieren" (bildungsspr., Fachspr.) und "divergierend" (bildungsspr., Math.) genannt. In anderen Fällen darf sicherlich wegen des heute allgemein relativ hohen Bildungsniveaus Nullmarkierung stehen. Auch hierfür gibt es Beispiele. Das DDUW zeichnet Lemmata wie „adäquat”, „äquivalent”, ,hineinprojizieren”, „pornographisch”, „rudimentär” u. v. a. konsequent als bildungssprachlich aus. Der Rechtschreibduden ver- 
zichtet ganz bewußt darauf. Für literarische Kontexte prädisponiert könnte auch das als gehoben apostrophierte Wortgut, das "bei feierlichen Anlässen und gelegentlich auch in der Literatur" verwendet wird (DDUW ${ }^{2} 1989$, S. 9), gelten und natürlich das dichterische, das in der Gegenwartssprache ohnehin weitgehend veraltet ist und im Rechtschreibduden, soweit es überhaupt ausgezeichnet ist, schon jetzt als gehoben markiert ist. Die Auszeichnung ungezwungen könnte all denjenigen Lemmata beigestellt werden, die in inoffiziellen, aber auch in offiziellen Kommunikationskontexten in ungezwungenem Sprachgebrauch verwendet werden bzw. verwendet werden können. Bei diesem Ansatz würde sich die Anzahl der unterschiedlichen stilistischen Markierungen in gewisser Weise reduzieren. Eine Reduzierung der stilistischen Marker auf nur drei wird letztlich aber nicht möglich sein, und dies schon deshalb nicht, weil derbes oder vulgäres Wortgut in einem Drei-StufenModell nicht wirklich einzuordnen ist. Allerdings würde bei Ludwig und in der vorgeführten Modifizierung seines Modells der unscharfe Begriff Umgangssprache und damit "das unselige ugs." (Wolski 1995, S. 354) obsolet werden. Die Markierung ungezwungen könnte auch ohne weiteres zu diatopischen Angaben hinzutreten, wenn sich dies als notwendig erweisen sollte.

Während Klaus-Dieter Ludwig letztlich für eine Reduzierung der stilistischen Auszeichnungen eintritt, plädiert Ulrich Püschel für ein „offenes Beschreibungsvokabular, das auch Ad-hoc-Beschreibungen zuläßt" (Püschel 1989, S. 698). Nun kann man Püschel zwar darin zustimmen, daß "die Nuancen im Wortgebrauch" eigentlich „umfassend und detailliert beschrieben werden" müBten (Püschel, ebenda). Aber sein Postulat, "der Hang zur ökonomischen Beschreibungsform" dürfe "nicht zu meist nichtssagenden Einwort-Kommentaren führen" (Püschel, ebenda), steht in einem deutlichen Gegensatz zu den Bedingungen und Anforderungen eines allgemeinen Gebrauchswörterbuches, zumal wenn es sich vorwiegend als Bedeutungswörterbuch versteht. Hier kann nur mit Hilfe stilistischer oder gebrauchsspezifischer Markierungen ein Hinweis auf den tendenziellen Gebrauchsrahmen eines Wortes gegeben werden, eine Art stark verkürzter Handlungsanweisung zum richtigen Sprachgebrauch, wie es weiter oben schon angedeutet wurde. Oder man müBte die Anzahl der im Wörterbuch verzeichneten Lemmata deutlich verringern, was aber meistens mit den Erwartungen der Benutzer kollidiert.

\subsection{Markierungskriterien und Markierungspraxis}

Es gibt in der Lexikographie keinen Konsens darüber, wieviele und welche Stilschichten oder Stilebenen sinnvollerweise eigentlich im Wörter- 
buch anzusetzen sind. Außerdem fallt auf, daß „die Zuordnung diastratischer Markierungen zu Wörtern bzw. einzelnen Verwendungsweisen lexikalischer Einheiten" zum Teil "erheblich" schwankt (Ludwig 1995, S. 289). Daraus kann man aber nun gerade nicht ableiten, daB es generell an Markierungskriterien fehle, auch wenn solche vielleicht nicht in jedem Wörterbuch befriedigend beschrieben sein mögen. Auch bei der stilistischen Bewertung lexikalischer Einheiten gilt, was man als ein $\mathrm{Zu}$ sammenspiel einer objektiven und einer subjektiven "Bestimmung" um diesen Begriff aus der Biologie heranzuziehen - beschreiben könnte. Die „objektive Bestimmung” erfolgt auf der Grundlage des Belegmaterials, das nicht nur gesammelt wird, um die bloße Existenz einer lexikalischen Einheit nachzuweisen. Einzelbeleganalyse und Belegvergleich, auch die Berücksichtigung der Textsorte, der ein bestimmter Quellentext zuzuordnen ist, ergeben oft auch Hinweise auf den stilistischen Wert der Einheit. Zur "objektiven Bestimmung" tritt die "subjektive" hinzu. Sie beruht auf dem intuitiven Wissen des kompetenten Sprechers um die "durch das NormbewuBtsein der Sprachgesellschaft gezogenen Grenzen zwischen Umgangssprache und gesprochener Standardsprache" (Steger 1984, S. 268). Wo Beleganalyse und Belegvergleich zur stilistischen Bestimmung einer Einheit nicht ausreichen, können entsprechende Zuordnungen oftmals nur durch "subjektive Bestimmung" erfolgen, wenn man einmal den Vergleich mit anderen Wörterbüchern unberücksichtigt läBt. Nun sind sprachliche Kompetenz und Sprachgefühl allerdings keine fixen Größen. Sie unterscheiden sich von Wörterbuchbearbeiter zu Wörterbuchbearbeiter, hängen von unterschiedlichen Faktoren ab wie Alter, Ausbildung, sozialer Zugehörigkeit und auch von regionaler Gebundenheit, wie Helmut Henne im Hinblick auf die stilschichtenspezifische Beurteilung von lexikalischen Einheiten im Vergleich zwischen ostdeutschen und westdeutschen Wörterbüchern feststellt (vgl. hierzu Henne 1988, S. 821). Auch das Normenbewuftsein verändert sich selbstverständlich, was sich gerade an der ganz unterschiedlichen Bewertung des zeitgenössischen Sprachgebrauchs in den Medien zeigt. Voneinander abweichende Markierungen sind also verständlich. Hausmann gesteht zu, daB selbst dann, „wenn ... die Markierung zwischen zwei Wörterbüchern in $40 \%$ der Fälle variiert”, dies „kein Argument für Verzicht auf Markierung” sei (Hausmann 1989, S. 650). 
3. Umgangssprachliche Lexik im Deutschen Universalwörterbuch

3.1 Die Auszeichnung ugs.

Die pragmatische Angabe umgangssprachlich (ugs.) steht im DDUW in rund 11.100 Fällen. Sie bezieht sich

a) auf das Stichwort selbst $\sim 3.900$ aalen, Betriebsnudel, kaltschnäuzig, löchern, Lulatsch, Irrsinnshitze, Pferdenatur, rausgehen, Schlamassel, verrammeln $\mathbf{u}$. a.

b) auf einzelne Lesarten, $\quad \sim 6.800$ alt (la: nicht alt werden Kontextbedeutungen oder feste Wendungen

c) auf morphologische Angaben

[nicht lange bleiben ...]) Holz (1:...viel Holz [eine große Menge von etw.])

Kummer (b: Schwierigkeit, mit der man nicht fertig wird]) Pelz (3: ${ }^{*}$ jmdm. auf den P. rücken etc.

[jmdm. mit einem Anliegen o. ä. zusetzen ...]) u. a.

a, A, anhauen, Beige, brauchen, Dorn, durchhauen, Einback, Joghurt, Junge u. a.

In der Mehrzahl der Fälle bezieht sich die Angabe ugs. auf Lesarten einzelner Lemmata. Wörter der Allgemeinsprache finden eine umgangssprachliche Umdeutung, die im Lexikon verzeichnet wird.

Wo sich die Angabe ugs. auf morphologische Kommentare bezieht, sind in der Regel Besonderheiten der Flexion (1) oder des Artikelgebrauchs (2) betroffen.

Beispiele zu (1): a, A ... das; - (ugs.: -s), - (ugs.: -s) ...

Beige, das; - (ugs.: -s) ...

Dorn, der; -[e]s, -en (ugs. auch: Dörner) ...

anhauen <unr. V.; hieb/(ugs.:) haute an, hat angehaven $>$... 
Beispiel zu (2) Joghurt, ... (ugs. u. österr.,

bes. wiener.) auch: die; $-\mathbf{s}-[\mathbf{s}]$...

Gelegentlich erfolgen auch Anmerkungen zur Syntax, so unter „brauchen (4)":

... er braucht heute nicht zu arbeiten/(ugs. auch ohne nzu":) braucht heute nicht arbeiten

Bei phonetischen Kommentaren findet sich der Marker ugs. nicht, was sich auch dadurch erklärt, daB das ausgewertete Quellenkorpus praktisch ausschlieBlich schriftliche Quellen umfaBt. Gesprochene Sprache scheint nur über ihre Verschriftung auf, wenn zum Beispiel in der $\mathrm{Li}$ teratur mündliche Rede zitiert wird.

In vielen Fällen steht die pragmatische Angabe ugs. jedoch nicht isoliert. Sie ist vielmehr häufig kombiniert mit Diafrequenzangaben und/oder diaevaluativen und/oder diatopischen Markern. In Tabelle 1 sind nur solche Fälle mit Anzahl der Fundstellen und Beispiellemmata aufgeführt, in denen sich die komplexe Angabe auf das Lemma bezieht. Im Hinblick auf die pragmatische Angabe wurden nur die Buchstaben $\mathrm{A}-\mathrm{K}$ durchsucht. Weitere Kombinationen können also auftreten, hätten dann aber mit großer Wahrscheinlichkeit als Einzelfälle zu gelten.

\begin{tabular}{|l|c|l|}
\hline Markierung & Anzahl & Beispiellemmata \\
\hline auch ugs. & 2 & aua, Streicheleinheit \\
\hline häufig ugs. & 1 & -mann \\
\hline sonst ugs. scherzh. & 6 & Gockel, Rempler, hieven \\
\hline ugs. abwertend od. gutmütig & 1 & Jäckel \\
\hline ugs. auch scherzh. & 1 & jawoll \\
\hline ugs., auch abwertend & 1 & Sozi \\
\hline ugs., bes. Sport Jargon & 1 & tricksen \\
\hline ugs. emotional & 132 & $\begin{array}{l}\text { durchschleppen, klatschnaB, } \\
\text { klapperdürr, pesen, ratzekahl, } \\
\text { Traumfrau }\end{array}$ \\
\hline ugs. emotional verstärkend & 108 & $\begin{array}{l}\text { blitzsauber, Bombenform, } \\
\text { Heidengeld, KlassefuBball, } \\
\text { Riesenhunger, stocksauer }\end{array}$ \\
\hline ugs., fam. & 2 & abknabbern, Zuckerpuppe \\
\hline ugs., häufig abwertend & 1 & Gewese \\
\hline ugs. iron. & 2 & museumsreif, oberschlau \\
\hline ugs.(,) leicht abwertend & 9 & $\begin{array}{l}\text { Allerweltsgesicht, Allerwelts- } \\
\text { geschmack, Operetten-, Plüsch, } \\
\text { Feld-Wald-und-Wiesen-, }\end{array}$ \\
\hline
\end{tabular}




\begin{tabular}{|c|c|c|}
\hline & & $\begin{array}{l}\text { Nullachtfünfzehn, } \\
\text { Schlafstadt }\end{array}$ \\
\hline ugs., meist abwertend & 25 & $\begin{array}{l}\text { Baulöwe, eindrillen, Ge- } \\
\text { stotter, Hampelei, Klein- } \\
\text { kram, Kocherei, muffelig, } \\
\text { treudeutsch }\end{array}$ \\
\hline ugs., meist scherzh. & 1 & Lustmolch \\
\hline ugs. $(, / ;)$ oft abwertend & 14 & $\begin{array}{l}\text { einpauken, Fachsimpelei, } \\
\text { FreBsack, säbeln, Schnippe- } \\
\text { lei, nachplappern, Wichtig- } \\
\text { tuer, wichtigtuerisch, } \\
\text { schniegeln, Schussel }\end{array}$ \\
\hline ugs., oft scherzh. & 14 & $\begin{array}{l}\text { bemopsen, Frānkli, Fressalien, } \\
\text { Gesichtskontrolle, Junggesel- } \\
\text { lenwirtschaft }\end{array}$ \\
\hline $\begin{array}{l}\text { ugs. }(, / ;) \text { oft scherzh. od. } \\
\text { abwertend }\end{array}$ & 4 & Oma, Opa, Kintopp \\
\hline $\begin{array}{l}\text { ugs., oft scherzh. od. } \\
\text { leicht abwertend }\end{array}$ & 1 & Landratte \\
\hline ugs. scherzh. & 219 & $\begin{array}{l}\text { Apparillo, Ballettratte, be- } \\
\text { duseln, beurgrunzen, erblonden, } \\
\text { Jubelgreis, Storchbein }\end{array}$ \\
\hline ugs. scherzh. abwertend & 1 & Flachland tiroler \\
\hline $\begin{array}{l}\text { ugs. scherzh.(,) oft } \\
\text { abwertend }\end{array}$ & 3 & $\begin{array}{l}\text { Kilometerfresser, Flimmer- } \\
\text { kiste, Iwan }\end{array}$ \\
\hline ugs.(,) selten & 4 & $\begin{array}{l}\text { beschwipsen, Hintere, } \\
\text { mörderlich, überwiegen, } \\
\text { [der Brief wiegt über] }\end{array}$ \\
\hline ugs. $(, / ;)$ seltener & 4 & $\begin{array}{l}\text { hineinrasseln, Hinterste, } \\
\text { Lapperei, nachtun }\end{array}$ \\
\hline ugs. spött(isch) & 12 & $\begin{array}{l}\text { Bierbauch, Biereifer, } \\
\text { ehrpusselig, Hintertupfingen, } \\
\text { Kleinkleckersdorf, } \\
\text { Pomadenhengst }\end{array}$ \\
\hline ugs., spöttisch abwertend & 1 & HasenfuB \\
\hline ugs. übertreibend & 1 & ohrenbetäubend \\
\hline ugs. veraltend & 2 & $\begin{array}{l}\text { Eckensteher, Franzmann, } \\
\text { Jägersmann, Jubelperser, } \\
\text { Mannsleute, Tippfräulein }\end{array}$ \\
\hline ugs. veraltend scherzh. & 2 & $\begin{array}{l}\text { Künstlermähne, Poussier- } \\
\text { stengel }\end{array}$ \\
\hline ugs. verhüll. & 4 & Engelmacherin, Hundertfūnf- \\
\hline
\end{tabular}




\begin{tabular}{|l|l|l|} 
& & undsiebziger, Scheibenkleister \\
\hline ugs. verhüll. scherzh. & 1 & Allerwerteste \\
\hline ugs. zum Ausdruck ... & 1 & basta \\
\hline veraltet, noch ugs. scherzh. & 1 & Festivität \\
\hline veraltend, sonst ugs. scherzh. & 2 & Schulmeister, Schulmeisterei \\
\hline veraltet ugs. abwertend & 1 & Itzig \\
\hline
\end{tabular}

Tabelle 1: Kombinationsvarianten von ugs. mit diahistorischen, diaevaluativen und Diafrequenzangaben

Nur auf Lesarten beziehen sich die kombinatorischen Angaben

bes. ugs.

meist ugs. scherzh.

ugs. drohend

ugs. intensivierend

ugs. meist iron.

ugs. scherzh. verhüll. frūher ugs. verhüll.

oft ugs.

ugs. emotional

bekräftigend

ugs., leicht abwertend, verhüll.

ugs., meist iron. abwertend

ugs. verstärkend
Jargon ugs.

oft ugs. abwertend

ugs. gelegentlich

abwertend

ugs., oft scherzh.

od. iron.

veraltend, noch ugs.

Nach diesem Verzeichnis gibt es im DDUW 58 Kombinationsmöglichkeiten des Markers ugs. mit diahistorischen, diaevaluativen und Diafrequenzangaben. Gemessen an der Gesamtzahl der Fundstellen, in denen sich ugs. auf das Lemma bezieht $(\sim 3.900)$, handelt es sich überwiegend um Einzelfalle. Einigermaße prominent sind nur die Kombinationen

$\begin{array}{lr}\text { ugs. scherzh. } & 219 \\ \text { ugs. emotional } & 132 \\ \text { ugs. emotional verstärkend } & 108 \\ \text { ugs., meist abwertend } & 25 \\ \text { ugs. veraltend } & 24 \\ \text { ugs., oft scherzh. } & 14 \\ \text { ugs. spöttisch } & 12\end{array}$

Alle anderen Kombinationen treten nur vereinzelt auf. Die Auszeichnungspraxis führt gelegentlich zu gewissen vordergründigen Inkonsequenzen. So gelten z. B. die Lemmata "klapperdürr", "klitschnaB" und "Traumfrau" als ugs. emotional, wohingegen "blitzsauber”, „Bombenform" und "Riesenhunger", die nach demselben Wortbildungsmuster gebildet sind, mit ugs. emotional verstärkend ausgezeichnet sind. Ähnliches gilt für "Schnippelei”, das als ugs., oft abwertend apostrophiert wird, und "Hampelei”, das ugs., meist abwertend sein soll. Solche Divergenzen sind in der Regel auf die unterschiedliche Beleglage zurückzuführen. Insofern können sie durchaus begründet sein. 


\subsection{Umgangssprache und regionale Gebundenheit}

Mit diatopischen Markern steht die pragmatische Angabe ugs. im DDUW in 31 Kombinationen (bezogen auf das Lemma, Tabelle 2):

\begin{tabular}{|c|c|c|}
\hline Markierung & Anzahl & Beispiellemmata \\
\hline bayr., ōsterr. ugs. & 25 & $\begin{array}{l}\text { Binkel, Grant, Herzbinkerl, } \\
\text { mollert, Radi, Schlapfen, } \\
\text { Watsche }\end{array}$ \\
\hline bes. berlin. ugs. & 1 & mittenmang \\
\hline bes. österr. ugs. & 5 & $\begin{array}{l}\text { allweil, durchwegs, Genierer, } \\
\text { Katzelmacher, Wichtigmacher }\end{array}$ \\
\hline bes. nordd. ugs. abwertend & 2 & Nöler, Nölerei \\
\hline bes. schweiz. ugs. & 1 & salü \\
\hline bes. südd., österr. ugs. & 3 & Lackel, Postler, pumpern \\
\hline $\begin{array}{l}\text { bes. südd., österr. ugs. } \\
\text { abwertend }\end{array}$ & 1 & Lackel \\
\hline $\begin{array}{l}\text { bes. sūdd., sonst ugs. } \\
\text { scherzh. }\end{array}$ & 1 & Gockel \\
\hline landsch., bes. nordd. ugs. & 2 & quackeln, Rummelplatz \\
\hline $\begin{array}{l}\text { landsch., bes. nordd. ugs. } \\
\text { abwertend }\end{array}$ & 1 & Salm \\
\hline landsch. ugs. & 35 & $\begin{array}{l}\text { Hudeler, hudeln, krengeln, } \\
\text { Murkel, pullen, rappelig, } \\
\text { runksen, Schnäppchen, } \\
\text { Simpel, Torkel, trapsen } \\
\end{array}$ \\
\hline landsch. ugs. abwertend. & 4 & $\begin{array}{l}\text { murkelig, Ruschel, } \\
\text { ruschelig, ruscheln }\end{array}$ \\
\hline landsch., österr. ugs. & 1 & nackert \\
\hline landsch., ugs. veraltend & 1 & Destille \\
\hline veraltet, noch landsch. ugs. & 1 & Hudel \\
\hline nordd. ugs. & 23 & $\begin{array}{l}\text { Buttje, Modder, muddeln, } \\
\text { pruschen, pulen, quesen, } \\
\text { sabbeln, tüterig, zackerieren }\end{array}$ \\
\hline nordd. ugs. abwertend & 6 & $\begin{array}{l}\text { nölen, nölig, prünen, } \\
\text { Quasse, quassig, Sabbel }\end{array}$ \\
\hline österr. ugs. & 142 & $\begin{array}{l}\text { Dutte, Hatscher, kiefeln, } \\
\text { Patscherl, Pracker, Schraufen }\end{array}$ \\
\hline österr. ugs. abwertend & 12 & blad, Blade, böhmakeln, \\
\hline
\end{tabular}




\begin{tabular}{|l|l|l|} 
& & Kepplerin, packeln \\
\hline österr. ugs., sonst veraltet & 1 & ausdeutschen \\
\hline österr., ugs. scherzh. & 1 & Alpendollar \\
\hline ostmd., südd., österr. ugs. & 2 & hintergehen, hinterbringen \\
\hline schweiz. ugs. & 6 & $\begin{array}{l}\text { geschupft, Klaus, nigelnagel- } \\
\text { neu, Pöstler, Töf }\end{array}$ \\
\hline südd. ugs. & 3 & $\begin{array}{l}\text { herumtun, strenzen, } \\
\text { unterschlupfen }\end{array}$ \\
\hline südd., österr. ugs. & 35 & $\begin{array}{l}\text { aufschnaufen, bissel, Buschen, } \\
\text { Busserl, drausbringen, } \\
\text { Grantigkeit, Hascherl }\end{array}$ \\
\hline ugs., bes. berlin. & 1 & Steppke \\
\hline ugs., bes. nordd. & 1 & abbleiben \\
\hline ugs., bes. ostmd. & 1 & behumpsen \\
\hline ugs., bes. sächsisch scherzh. & 1 & Blümchenkaffee \\
\hline ugs., bes. südd. & 1 & radeln \\
\hline ugs., bes. südd., österr. & 3 & Kraxelei, kraxeln, Mannsbild \\
\hline
\end{tabular}

Tabelle 2: Kombinationsvarianten von ugs. mit diatopischen Markern im DDUW

Regionale Gebundenheit umgangssprachlicher lexikalischer Einheiten wird in den Standardwörterbüchern der deutschen Gegenwartssprache angezeigt, gemessen am dokumentierten Ausschnitt aus dem Gesamtwortschatz allerdings nur in einem bescheidenen Maße. Immer bezogen auf das Lemma selbst und nicht auf einzelne Lesarten sind im DDUW 323 Einträge als areal fixiert und zusätzlich als umgangssprachlich ausgezeichnet. Das sind knapp 0,27\%. Nur bezogen auf die als umgangssprachlich markierten Lemmata sind es knapp 8,3\%, dabei sind auch diejenigen Fälle berücksichtigt, die Austriazismen und Helvetismen betreffen. Als Kommentare zu Lesarten kommen, dies sei der Vollständigkeit halber ergänzt, die Kombinationsvarianten berlin. ugs.; ugs., bes. bayr.; ugs. landsch. und ugs. scherzh., bes. nordd. hinzu.

Vergleicht man nun unterschiedliche Wörterbücher, ist festzustellen, daß auch hinsichtlich der Dokumentation regionaler Gebundenheit umgangssprachlicher Lexik Divergenzen auftreten. Zur Unsicherheit im Hinblick auf die gebrauchs- oder situationsspezifische Markierung tritt hier die Unsicherheit im Hinblick auf die areale Verbreitung einzelner Lexeme hinzu. Dies zeigt die Übersicht in Tabelle 3 an Hand einiger Beispiele $(\emptyset$ = Nullmarkierung; "-" steht für fehlenden Eintrag im Wörterbuch). 


\begin{tabular}{|c|c|c|c|c|c|c|c|c|}
\hline $\mid$ & 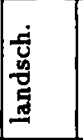 & 1 & 1 & 离 & 1 & 1 & 11 & 1 \\
\hline $\mid$ & 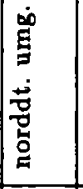 & 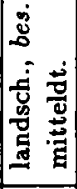 & 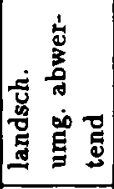 & ) & 1 & 1 & 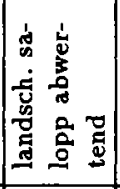 & 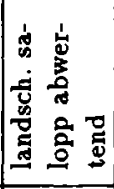 \\
\hline 3 & $\dot{\infty}$ & 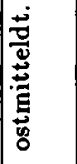 & 递 & 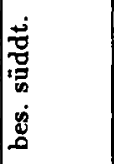 & 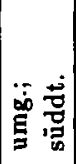 & 1 & 1 & $\theta$ \\
\hline 方 & है & 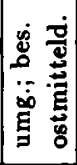 & 宽 & 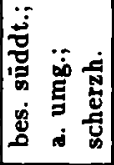 & $\ddot{\text { है }}$ & $\theta$ & 客 & 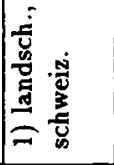 \\
\hline$\vec{z}$ & 1 & 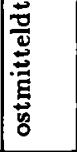 & 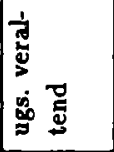 & 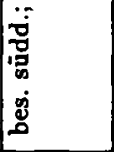 & 1 & 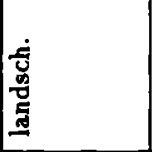 & 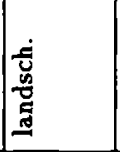 & 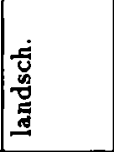 \\
\hline $\mid \begin{array}{l}\infty \\
\\
0\end{array}$ & 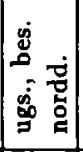 & 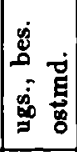 & 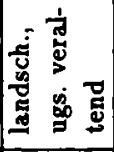 & 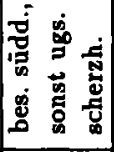 & $\begin{array}{l}0 \\
00 \\
9 \\
8 \\
8 \\
0\end{array}$ & 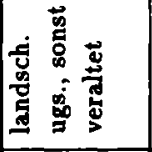 & 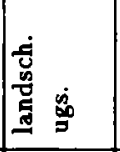 & 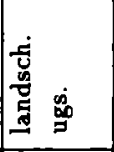 \\
\hline 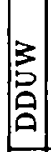 & 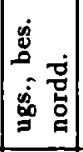 & 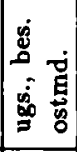 & 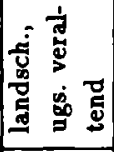 & 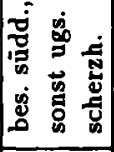 & $\begin{array}{l}0 \\
g \\
0 \\
0 \\
0\end{array}$ & 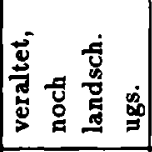 & 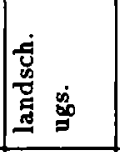 & 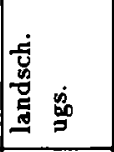 \\
\hline : & 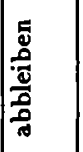 & 焉 & 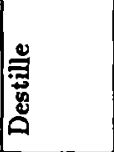 & 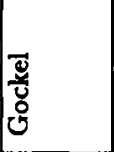 & E & 䄈 & 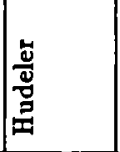 & 형 \\
\hline
\end{tabular}




\begin{tabular}{|c|c|c|c|c|c|c|c|c|c|c|}
\hline 1 & 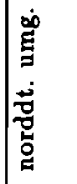 & 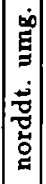 & 1 & 1 & 1 & 1 & 1 & 1 & 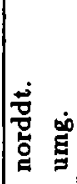 & 竎 \\
\hline 1 & 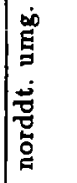 & 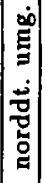 & 1 & 1 & 1 & 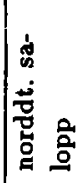 & 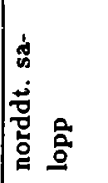 & 1 & 苞 & 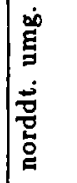 \\
\hline$Q$ & 窎 & $\mid$ & نَ & 1 & 1 & 㭶 & 1 & 1 & 1 & 菅 \\
\hline 1 & 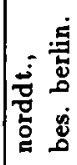 & 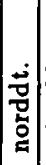 & 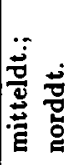 & 突 & 1 & 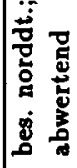 & 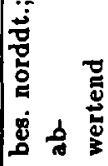 & 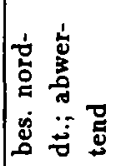 & 苞 & 荤 \\
\hline 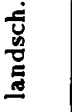 & 营 & $\begin{array}{l}\overrightarrow{\mathrm{g}} \\
\mathrm{D} \\
\mathrm{o} \\
\mathrm{g}\end{array}$ & 1 & 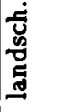 & 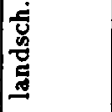 & Dृ & 1 & 1 & 1 & 1 \\
\hline 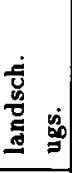 & 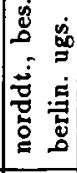 & $\mid \begin{array}{l}\dot{0} \\
0 \\
0 \\
\dot{g} \\
\dot{z} \\
0 \\
0\end{array}$ & 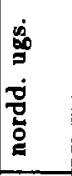 & 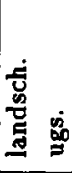 & 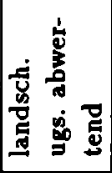 & 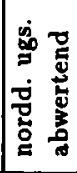 & 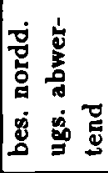 & 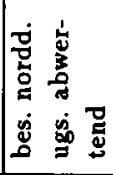 & $\begin{array}{l}\dot{0} \\
\dot{\theta} \\
\dot{0} \\
\dot{\vec{g}} \\
\dot{0} \\
\dot{g}\end{array}$ & 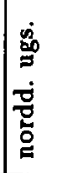 \\
\hline 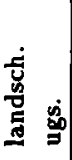 & $\begin{array}{ll}\dot{\Xi} & \\
\dot{\Xi} & \\
0 & \\
\dot{8} & \dot{0} \\
\dot{0} & 0\end{array}$ & $\begin{array}{c}\dot{0} \\
0 \\
= \\
\dot{0} \\
\overline{0} \\
0 \\
0\end{array}$ & $\begin{array}{l}\dot{0} \\
0 \\
0 \\
0 \\
0 \\
0 \\
0 \\
0\end{array}$ & 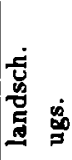 & 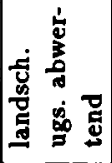 & 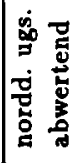 & 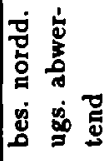 & 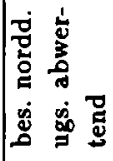 & 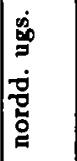 & 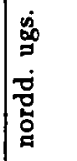 \\
\hline 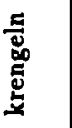 & 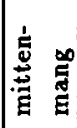 & 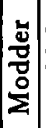 & 몰 & $\frac{\bar{d}}{3}$ & $\frac{\infty}{3}$ & 동 & $\frac{2}{\frac{2}{2}}$ & $\mid \begin{array}{l}\overline{0} \\
\frac{0}{20} \\
z\end{array}$ & 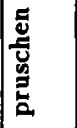 & $\frac{\text { E⿱ }}{\vec{Z}}$ \\
\hline
\end{tabular}




\begin{tabular}{|c|c|c|c|c|c|c|c|c|c|}
\hline & 1 & 1 & 离 & 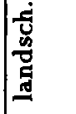 & 1 & 1 & 1 & 1 & 1 \\
\hline 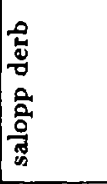 & 1 & 1 & 敛 & 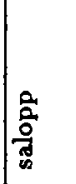 & 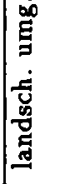 & 1 & 1 & 1 & 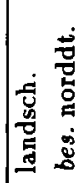 \\
\hline$\stackrel{\infty}{\Xi}$ & 1 & 苞 & $\dot{0}$ & है & 首 & 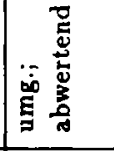 & $\stackrel{\dot{0}}{\Xi}$ & $\stackrel{\infty}{\Xi}$ & 足 \\
\hline 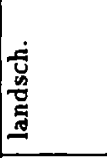 & 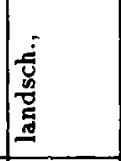 & ن. & E் & 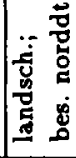 & E & 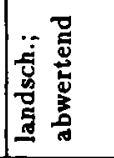 & 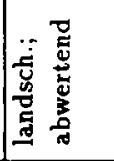 & 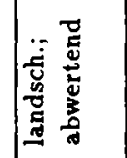 & 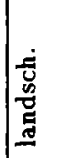 \\
\hline 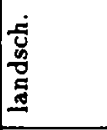 & 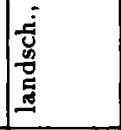 & نे & $\dot{0}$ & 离 & 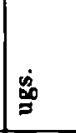 & 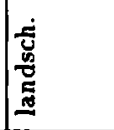 & 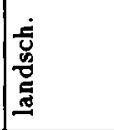 & 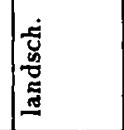 & 总 \\
\hline 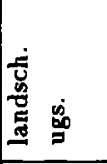 & 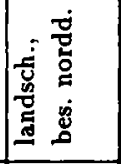 & 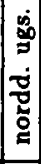 & 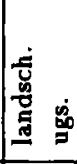 & 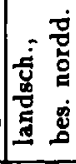 & 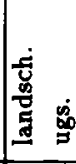 & 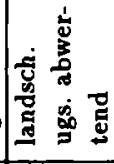 & 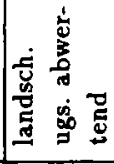 & 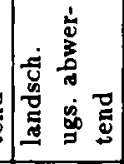 & 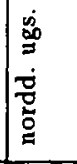 \\
\hline 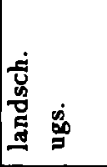 & 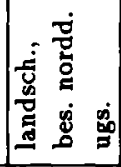 & \begin{tabular}{|c|}
0 \\
0 \\
0 \\
0 \\
0 \\
0 \\
0
\end{tabular} & 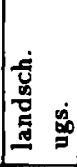 & 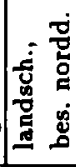 & 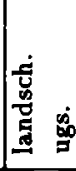 & 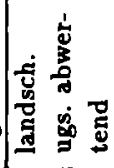 & 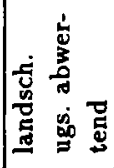 & 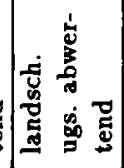 & 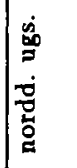 \\
\hline 袁 & 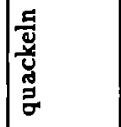 & 豙 & $\mid$ & 宔 & 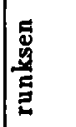 & 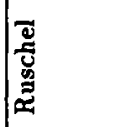 & 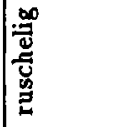 & 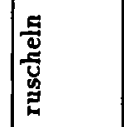 & 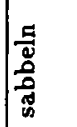 \\
\hline
\end{tabular}




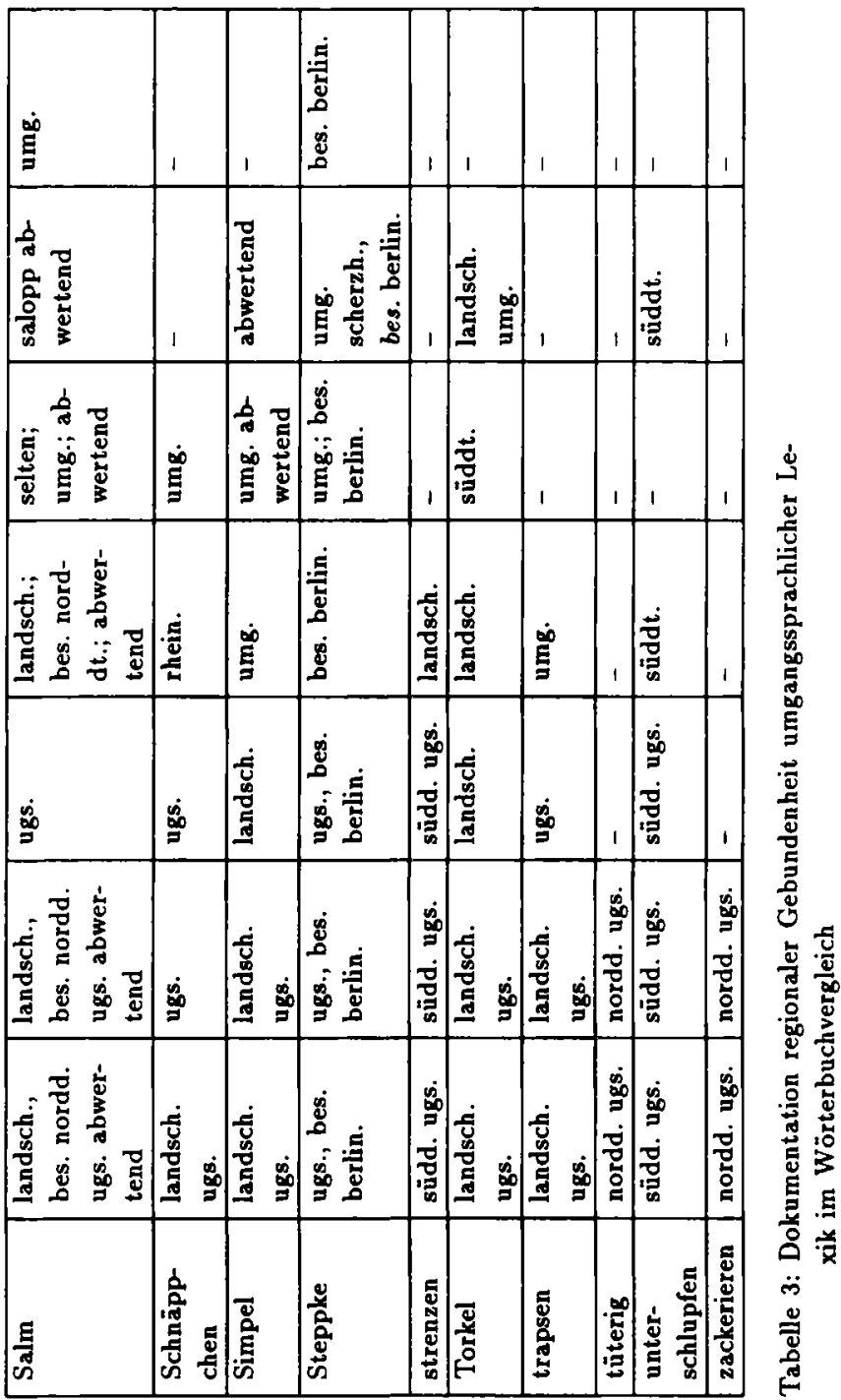


Bei der näheren Analyse dieser Aufstellung zeigt sich, daB zum Beispiel das Stichwort "Rummelplatz" im DDUW und im GWB als landschaftl. (d. h., das areale Geltungsgebiet läßt sich nicht näher eingrenzen), bes. nordd. ugs. ausgezeichnet ist, wobei nicht ganz eindeutig erkennbar ist, ob sich das Etikett ugs. nur auf nordd. oder auch auf landsch. bezieht. Der Rechtschreibduden (Duden 1) reduziert diese Angabe auf den Marker ugs. und verzichtet darauf, den räumlichen Geltungsbereich von "Rummelplatz” auszuweisen. „Rummelplatz” wird damit zu einem Wort der Allgemeinsprache. Brockhaus-Wahrig zeichnet das Lexem als landsch.; bes. norddt. aus, ohne dab eine stilistische Wertung erfolgt. Das einbändige Wahrig-Wörterbuch steht zum Rechtschreibduden und beschränkt sich ebenfalls auf das Etikett umg. Auffallig sind auch die Abweichungen zwischen dem Wörterbuch der deutschen Gegenwartssprache (WdG) und dem Handwörterbuch der deutschen Gegenwartssprache (HWdG). Im ersten ist „Rummelplatz” salopp, im zweiten landsch.

In anderen Fällen sind die Unterschiede zwischen den einzelnen Wörterbüchern weniger markant. DDUW und GWB zeichnen das Lemma "rappelig" als landsch. ugs. aus, während Duden 1, BrockhausWahrig, der einbändige Wahrig und das HWdG einheitlich die Markierung umgangssprachlich aufweisen. Nur das WdG durchbricht die Reihe, indem es den Marker salopp setzt. Insgesamt ist bei den untersuchten Lemmata festzustellen, daB die Abweichungen bei kombinierten diatopischen und stilistischen Angaben doch relativ gering sind. In vielen Fälle beruhen sie allein darauf, daß die bei der diatopischen Angabe stehende stilistische einmal entfällt oder auch umgekehrt. "Murkel” (= kleines Kind) gilt im DDUW und GWB zum Beispiel als landsch. ugs., während es Duden 1 und Brockhaus-Wahrig nur als landsch. markieren. Umgekehrt bei "Schnäppchen”. Während das DDUW das Stichwort noch als landsch. ugs. auszeichnet, schlagen es GWB, Duden 1 und das einbändige Wahrig-Wörterbuch einhellig der Umgangssprache zu. Dies entspricht dem heutigen Sprachgebrauch, soweit er durch die Dudenkartei belegt ist. Hier finden sich Belege aus dem „Spiegel” (32/1989, S. 91), dem „Mannheimer Morgen” (13.12.1985, S. 29), der "ADAC motorwelt" (z. B. 11/1986, S. 24), einer der auflagenstärksten Zeitschriften im deutschsprachigen Raum mit entsprechend flächendeckender Verbreitung, aus der "Welt” (26.7.1977, S. 20), der "Süddeutschen Zeitung” (11.12.1981, S. 174) und dem „Flensburger Tageblatt” (Ostern 1984, S. 20), neben denen die Belege aus dem "Kölner Wochenspiegel” $(2.1 .1985$, o. S.) und der ebenfalls in Köln erscheinenden Zeitschrift "Express” (12.5.1984, S. 11) kaum auffallen. Die Beleglage spricht also nicht für eine räumlich begrenzte Verbreitung dieses Wortes. Insofern 
stellt die Auszeichnung rhein. im Brockhaus-Wahrig einen Ausreißer dar. Sie wird dem tatsächlichen Sprachgebrauch nicht mehr gerecht.

Wie erklären sich nun derartige Abweichungen? Zum einen ist es bei einem Wörterbuchvergleich nicht ganz unwichtig, die Erscheinungsjahre der verglichenen Wörterbücher zu berücksichtigen. So liegt 2 wischen dem Erscheinen des letzten Bandes des Deutschen Wörterbuches von Brockhaus-Wahrig und dem Erscheinen des letzten Bandes der zweiten Auflage des Großen Wörterbuchs der deutschen Sprache über ein Jahrzehnt, was gerade hinsichtlich der rasanten Entwicklungen innerhalb des Wortschatzes eine nicht unerhebliche Zeit ist. Zum anderen hängt die Etikettierung der Wörterbucheinträge von der schon oben erwähnten sprachlichen Kompetenz der unterschiedlichen Wörterbuchbearbeiter $\mathrm{ab}$, die zum Teil auch von individuellen, außersprachlichen Faktoren bestimmt ist. Hier hilft in der Praxis zwar die Abstimmung im Bearbeiterkreis in gewisser Weise aus dem Dilemma; echte Klarheit ist aber nicht zu erreichen. Klaus-Dieter Ludwig geht in diesem Punkt sogar soweit zu sagen, daB es gerade in bezug auf die „stilistische Bewertung und Kennzeichnung von lexikalischen Einheiten im Wörterbuch" keine „endgültige, alle Wörterbuchbenutzer und Linguisten befriedigende Lösung" geben werde (Ludwig 1995, S. 229). Man darf auch nicht ganz übersehen, daB beim Vergleich von diasystematischen Angaben in Wörterbüchern auch der Wörterbuchtyp berücksichtigt werden muB. Diasystematische Angaben - und hier eben ganz besonders die stilistischen und die diatopischen - können in relativ umfangreichen Bedeutungswörterbüchern wie dem DDUW oder dem GWB komplexer ausfallen, die pragmatische Beschreibung des Lexems oder der Lesart kann exakter sein als zum Beispiel in einem Rechtschreibwörterbuch, das ja in erster Linie orthographische Informationen vermitteln will und alle Zusatzinformationen entsprechend rudimentär hält. So wurde bei der Bearbeitung der 20. Auflage des Rechtschreibdudens ganz bewuBt eine Reduzierung kombinatorischer pragmatischer Angaben vorgenommen, was in einigen Fällen den Verzicht auf zusätzliche stilistische Angaben bei landschaftlichem Wortgut bedeutete. Steht bei "murkelig” im DDUW und im GWB die Markierung landsch. ugs. abwertend, so steht im Rechtschreibduden nur landsch. Damit ist natürlich auch der Tatsache Rechnung getragen, daB auf der Grundlage des Belegmaterials und der Forschung stilistische Nuancierungen gerade bei landschaftlichem Wortgut nicht immer leicht nachzuweisen sind, und gröBere Befragungen hierzu scheiden schon aus praktischen Gründen aus. Es ist also durchaus gerechtfertigt, einerseits intuitive, andererseits zum Teil hoch komplexe und damit vom Wörterbuchbenutzer nicht immer leicht zu dechiffrierende pragmatische Angaben zu vereinfachen. Das ändert jedoch nichts an der Tatsache, „daB 
die traditionellen Marker zur Kennzeichnung des Stellenwerts" eines Lexems "genauer definiert und besser aufeinander bezogen sein müssen", wie Ludwig fordert (Ludwig 1991, S. 230). Diese Forderung ist jedoch nicht allein von der praktischen Lexikographie zu erfüllen. Sie braucht hierzu Grundlagen, die von der Sprachforschung und insbesondere der Stilforschung noch zu präzisieren wären.

\section{Literatur}

a) Nachschlagewerke

Brockhaus-Enzyklopädie in 24 Bänden. 19., völlig neubearbeitete Auflage. Band 22. Mannheim 1993.

Brockhaus-Wahrig - Deutsches Wörterbuch in sechs Bānden. Herausgegeben von Wahrig, Gerhard/Krämer, Hildegard/Zimmermann, Harald. Wiesbaden und Stuttgart $1980 \mathrm{ff}$.

Das Wörterbuch der deutschen Gegenwartssprache. Herausgegeben von Ruth Klappenbach und Wolfgang Steinitz. 9., bearbeitete Auflage. Berlin $1978 \mathrm{ff}$.

Duden - Das große Wörterbuch der deutschen Sprache in acht Bänden. 2., völlig neu bearbeitete und stark erweiterte Auflage. Herausgegeben und bearbeitet vom Wissenschaftlichen Rat und den Mitarbeitern der Dudenredaktion unter der Leitung von Günther Drosdowski. Mannheim $1993 \mathrm{ff}$.

Duden - Deutsches Universalwörterbuch. 2., völlig neu bearbeitete und stark erweiterte Auflage. Herausgegeben und bearbeitet vom Wissenschaftlichen Rat und den Mitarbeitern der Dudenredaktion unter der Leitung von Günther Drosdowski. Mannheim 1989.

Duden - Grammatik der deutschen Gegenwartssprache (Duden Bd. 4). 5., völlig neu bearbeitete und erweiterte Auflage. Herausgegeben und bearbeitet von Günther Drosdowski in Zusammenarbeit mit Eisenberg, Peter/Gelhaus, Hermann/Henne, Helmut/Sitta, Horst/Wellmann Hans. Mannheim 1995.

Duden - Rechtschreibung der deutschen Sprache (Duden Bd. 1). 20., völlig neu bearbeitete und erweiterte Auflage. Herausgegeben von der Dudenredaktion. Mannheim 1991.

Handwōrterbuch der deutschen Gegenwartssprache. Von einem Autorenkollektiv unter der Leitung von Günter Kempcke. 2 Bde. Berlin 1984. 


\section{b) Sekundärliteratur}

Bichel, Ulf (1973): Problem und Begriff der Umgangssprache in der germanistischen Forschung. Tübingen.

Bichel, Ulf (1980): Umgangssprache. In: Althaus, Hans-Peter/Henne, Helmut/Wiegand, Herbert Ernst (Hg.): Lexikon der Germanistischen Linguistik. 2., vollstāndig neu bearbeitete und erweiterte Auflage (Studienausgabe II). Tübingen. S. 379-383.

BuBmann, Hadumod (1990): Lexikon der Sprachwissenschaft. 2. Auflage. Stuttgart.

Hausmann, Franz-Josef (1989): Die Markierung im allgemeinen einsprachigen Wörterbuch: eine Übersicht. In: Wörterbücher, S. 649 ff.

Henne, Helmut (1988): Stichwort Umgangssprache. Werkstattbericht zum neuen 'Paul'. In: Munske, Horst Haider u. a. (Hg.): Deutscher Wortschatz. Lexikologische Studien. Berlin/New York. S. 525 ff.

Lewandowski, Theodor (1976): Linguistisches Wörterbuch. 2., durchgesehene und erweiterte Auflage. Heidelberg.

Ludwig, Klaus-Dieter (1991): Markierungen im allgemeinen einsprachigen Wörterbuch des Deutschen. Ein Beitrag zur Metalexikographie. Tübingen (= Lexicographica Series Maior 38).

Ludwig, Klaus Dieter (1995): Stilkennzeichnungen und Stilbewertungen im deutschen Wörterbuch der Gegenwart. In: Stilfragen. Hg. von Gerhard Stickel. Berlin. S. 280 ff.

Lühr, Rosemarie (1990): Neuhochdeutsch. 3. Auflage. München.

Püschel, Ulrich (1989): Diaevaluative Markierungen im allgemeinen einsprachigen Wörterbuch. In: Wörterbücher, S. $693 \mathrm{f}$.

Ratke, Ingulf (1973): Die Umgangssprache. In: Muttersprache 83, S. $161 \mathrm{ff}$.

Steger, Hugo (1984): Bemerkungen zum Problem der Umgangssprache. In: Besch, Werner u. a. (Hg.): Festschrift für Siegfried Grosse zum 60. Geburtstag. Göppingen (= Göppinger Arbeiten zur Germanistik 423).

Steger, Hugo (1988): Erscheinungsformen der deutschen Sprache. „Alltagssprache” - „Fachsprache” - „Standardsprache” - „Dialekt” und andere Gliederungstermini. In: Deutsche Sprache, Heft 4, S. $289 \mathrm{ff}$.

Wiegand, Herbert Ernst (1989): Die deutsche Lexikographie der Gegenwart. In: Wörterbücher, S. $2100 f$.

Wörterbücher. Ein internationales Handbuch zur Lexikographie. Herausgegeben von Franz Josef Hausmann et alii. Berlin/New York 1989. (= Handbücher zur Sprach- und Kommunikationswissenschaft. Herausgegeben von Hugo Steger und Herbert Ernst Wiegand. Bde. 5.1 und 5.2).

Wolski, Werner (1995): Umgangssprachliches und Saloppes ohne Gewāhr. Anmerkungen zu einem neuen deutsch-russischen Wörterbuch. In: Zeitschrift für germanistische Linguistik 32.2, S. 342 ff. 